Portland State University

PDXScholar

\title{
A Novel Methodology for Spatial Damage Detection and Imaging Using a Distributed Carbon Nanotube- Based Composite Sensor Combined with Electrical Impedance Tomography
}

Hongbo Dai

University of Delaware

Gerard J. Gallo

University of Delaware

Thomas Schumacher

Portland State University, thomas.schumacher@pdx.edu

Erik T. Thostenson

University of Delaware

Follow this and additional works at: https://pdxscholar.library.pdx.edu/cengin_fac

Part of the Civil and Environmental Engineering Commons, and the Condensed Matter Physics

Commons

Let us know how access to this document benefits you.

\section{Citation Details}

Published as: Dai, H., Gallo, G.J., Schumacher, T. et al. A Novel Methodology for Spatial Damage Detection and Imaging Using a Distributed Carbon Nanotube-Based Composite Sensor Combined with Electrical Impedance Tomography. J Nondestruct Eval 35, 26 (2016).

This Pre-Print is brought to you for free and open access. It has been accepted for inclusion in Civil and Environmental Engineering Faculty Publications and Presentations by an authorized administrator of PDXScholar. Please contact us if we can make this document more accessible: pdxscholar@pdx.edu. 


\title{
A Novel Methodology for Spatial Damage Detection and Imaging \\ Using a Distributed Carbon Nanotube-based Composite Sensor Combined with Electrical Impedance Tomography
}

\author{
Hongbo Dai ${ }^{1,5, *}$, Gerard J. Gallo ${ }^{2,5}$, Thomas Schumacher ${ }^{3,}$, and Erik T. Thostenson ${ }^{2,4,5}$ \\ ${ }^{1}$ Civil and Environmental Engineering, University of Delaware, Newark, DE 19716, USA \\ ${ }^{2}$ Mechanical Engineering, University of Delaware, Newark, DE, 19716, USA \\ ${ }^{3}$ Civil and Environmental Engineering, Portland State University, Portland, OR, 97201, USA \\ ${ }^{4}$ Materials Science \& Engineering, University of Delaware, Newark, DE 19716, USA \\ ${ }^{5}$ Center for Composite Materials, University of Delaware, Newark, DE, 19716, USA \\ ${ }^{*}$ Corresponding author, e-mail address: hongbo@udel.edu (H.D.)
}

\begin{abstract}
This paper describes a novel non-destructive evaluation methodology for imaging of damage in composite materials using the electrical impedance tomography (EIT) technique applied to a distributed carbon nanotube-based sensor. The sensor consists of a nonwoven aramid fabric, which was first coated with nanotubes using a solution casting approach and then infused with epoxy resin through the vacuum assisted resin transfer molding technique. Finally, this composite sensor is cured to become a mechanically-robust, electromechanically-sensitive, and highly customizable distributed two-dimensional sensor which can be adhered to virtually any substrate. By assuming that damage on the sensor directly affects its conductivity, a difference imaging-based EIT algorithm was implemented and tailored to offer two-dimensional maps of conductivity changes, from which damage location and size can be estimated. The reconstruction is based on a newly defined adjacent current-voltage measurement scheme associated with 32 electrodes located along the boundary of the sensor. In this paper, we evaluate our methodology first by introducing well-defined damage where sections are either removed or narrow cuts are made on a series of sensor specimens. Finally, a more realistic damage scenario was investigated to show the capability of our methodology to detect impact damage on a composite laminate.
\end{abstract}


The resulting EIT maps are compared to visual inspection and thermograms taken with an infrared camera.

Keywords: Distributed sensing, carbon nanotube, composite materials, nonwoven fabric, electrical impedance tomography, non-destructive evaluation, damage detection, difference imaging.

\section{Introduction}

\subsection{Damage Sensing Approaches using Resistive Sensors}

Recent advances in composite materials have taken advantage of both nanotechnology and composite engineering and created a new era for developing novel and multifunctional composite sensors that possess high sensitivity and excellent mechanical response [1, 2] which are suitable for non-destructive evaluation (NDE) and structural health monitoring (SHM) applications. A number of researchers have validated the feasibility and sensitivity of employing carbon nanotube (CNT)-based nanocomposites as strain/damage sensors [3-9]. One approach to locate and image damage on a structure is by using a series of one-dimensional measurements collected from a two-dimensional (2-D) sensing area covered by densely-spaced strain gages [10] or from the quasi-distributed surface electrodes on an electrically conductive composite panel [11]. However, this quasi-2-D algorithm compromises the results by confining the possible damage locations to the grid points of the measurements. Electrical impedance tomography (EIT) is a true 2-D algorithm and has been recently studied to perform spatial damage evaluation on fiber reinforced composite plates [12-16], nanocomposites [17-19] and concrete members [20-23]. Nevertheless, the results from these studies are influenced by the anisotropic conductivity of the sensors used such that relatively low resolution and high background noise are observed in the final EIT maps [12-16, 19]. Additionally, field applications may be considerably limited due to the low sensitivity of the sensor, vulnerability to environmental effects $[17,21,22]$, and also fabrication challenges $[13-15,17,18]$.

In this study, a novel CNT-based composite sensor was first fabricated following a two-step method where CNTs are depositing from an aqueous dispersion onto a non-woven 
fabric followed infusion of epoxy resin $[9,24]$. The nanotube composite sensor can be adhered to virtually any shape to detect deformation and damage and is also mechanically robust and electrically isotropic. Electrodes are then attached to the sensor to allow measurement of the resistance changes in the CNT network and map local conductivity changes. Moreover, the manufacturing approach is inexpensive and can be readily scaled-up for large engineering applications. This paper demonstrates for the first time the feasibility of performing the difference imaging-based EIT on a CNT-based nonwoven composite sensor to estimate the location and severity of different types of damage.

\subsection{Carbon Nanotube-Based Composites as Sensors}

CNT-based composites are strong candidates for serving as distributed strain and damage sensors due to their extraordinary piezoresistivity [25], durability [26], and application versatility [27]. To date, a number of experimental studies have been performed on investigating and characterizing the electromechanical behavior of CNT-based nanocomposites and demonstrated their capability as in situ damage sensors [3]. For example, Gao et al. [4,6] have analyzed the electromechanical response of the CNT-based composite laminates subjected to static and cyclic loading conditions. The bulk resistance measured between the two ends of the nanotube composite specimen displayed a strong correlation with the applied load in real-time and identified the formation of damage in the composites through sudden increases in the electrical resistance due to damage-induced changes in the nanotube network. In addition, many applications that use CNT-based nanocomposites for SHM sensors have been documented such as damage sensing for composite joints [7,8], dynamic strain sensing on a small cantilever beam [5], integrated strengthening and monitoring of a concrete beam [28], wireless damage sensing of a small concrete beam [29], and dynamic strain monitoring of a full-scale reinforced concrete beam [30].

Due to the nanoscale size of CNTs combined with their large aspect ratio (length/diameter), an electrically conductive network can be established in CNT-based composites by integrating small amounts of CNTs by coating a carrier fabric [9]. This fabric can be either structural [28] or non-structural [9] to form an integrated strengthening/monitoring composite or a distributed sensor, respectively. The deposition of CNTs onto the carrier fabric 
can be achieved in several ways. The most important approaches include: coating the fabric with CNT-based sizing [9, 31], infusion of epoxy with dispersed CNTs into fabric [4, 6], and by electrophoretic deposition from a CNT solution $[32,33]$. In a recent study we have introduced a sizing approach and characterization of CNT-infused nonwoven fabrics as strain sensors [9]. This nonwoven fabric is a sheet of randomly-oriented aramid fibers with high open porosity (about 90\%). Due to the random, nonwoven structure the electrical conductivity of the resulting composite is isotropic. The sensor is piezoresistive, enabling the sensing of strain [9]. To determine the location of damage within the sensor multiple electrodes can be employed along with more advanced data analysis approaches, such as electric impedance tomography (EIT).

\subsection{Electrical Impedance Tomography}

Electrical impedance tomography has been extensively studied for medical and geological applications since the 1980s [34,35] but has been largely overlooked by the NDE and SHM communities until recently. By measuring boundary voltages EIT is able to map the internal conductivity distribution in an electrically conductive material [36-38]. In order to solve this non-linear and ill-posed inverse problem, regularization and linearization techniques as well as numerical solvers are employed to produce an approximate solution [36, 37]. Baltopoulos, et al. [12] used a 20-electrode EIT scheme to assess damage in carbon fiber reinforced composite laminates that were subjected to indentation impacts. The reconstructed conductivity maps were produced using the least-squares method with Tikhonov regularization and showed the localized areas with reduced conductivity corresponding to impact damage. Following the same EIT algorithm they also performed impact damage evaluation on a CNT-modified glass fiber-reinforced composite plate [13]. However, the resulting EIT maps from both studies showed a significant amount of artifacts and low resolution.

Hou and co-workers [17] first employed the EIT technique on a nanocomposite (i.e., CNT-PSS/PVA thin film) for spatial damage sensing. They created a small $(25 \mathrm{~mm} \times 25 \mathrm{~mm})$ planar sensor and used Gauss-Newton regularization algorithm for EIT imaging of etching damage. Loyola, et al. [14, 15] used EIT for strain and impact damage monitoring of glass fiber composites by implementing a CNT-based polymer thin film as spatial sensor. In these two experimental studies, a one-step linear reconstruction algorithm was employed and tuned to 
achieve normalized differential conductivity maps with optimal resolution. In addition, Loh, and co-workers [18] and Hou and Lynch [20] quantitatively correlated the reconstructed EIT maps with strain. A linear relationship was observed between the contrast of the reconstructed conductivity maps and strain. Some EIT applications have also been conducted to image surface cracking of concrete members [21] and to detect internal anomalies [23]. Recently, Hallaji, et al. [22] performed EIT on a silver paint sensor using an absolute imaging scheme to detect cracking of a concrete beam under three-point bending obtaining high-resolution quantitative reconstruction maps, which accurately show the complex and evolving crack pattern. Additionally, Tallman and coworkers used the difference imaging algorithm and conducted EIT on the carbon nanofiber epoxy plates [19] and carbon black filled glass fiber/epoxy laminates [16] for damage detection. They also performed the same EIT algorithm on the flexible carbon nanofiber/polyurethane composites [39] for imaging tactile contacts and distributed strains.

\section{EIT Methodology}

In order to generate EIT reconstructions of conductivity change it is required that a forward and inverse problem be solved. Figure 1 illustrates the reconstruction approach for mapping the sensor's internal distribution of conductivity change. The inputs and variables shown in the figure are defined in the following sections detailing the theoretical basis for the EIT methodology.

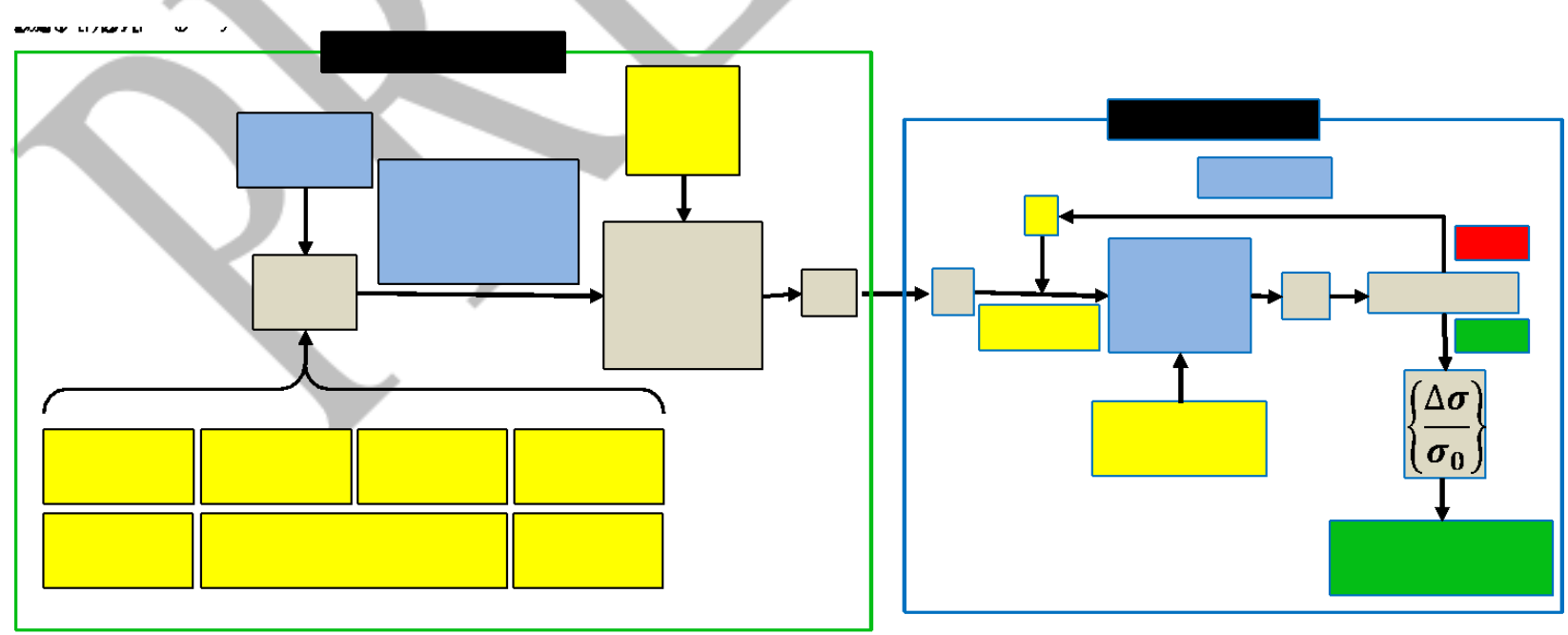


Figure 1. Flowchart visualizing EIT methodology implemented in this study.

\subsection{Forward Problem}

The forward problem solves the physical model for simulating the boundary voltages due to the applied electrical current in the 2-D sensor (see Figure 2 (a) and (b)) with the given geometry, boundary conditions, and assumed conductivity distribution of the sensing medium. The relationship between the conductivity $(\sigma)$ distribution inside a linear isotropic domain $(\Omega)$ with a smooth boundary $(\partial \Omega)$ and the boundary voltages $(u)$ is governed by a Laplacian equation derived from Maxwell's equations [37], assuming the absence of an interior current source [36]:

$$
\nabla \cdot(\sigma \nabla u)=0 \text {, in }(2-\mathrm{D} \Omega)
$$

In this study, the so-called complete electrode model (CEM) is used to apply the boundary conditions to the above governing equation. As extensively detailed in literatures $[36,37,40,41]$, CEM mainly defines the total amount of current and voltage at boundary electrodes and for the rest of the domain. CEM considers both the shunting effect and the contact impedance for each of the electrodes $[36,37,41]$. In addition, the finite element (FE) method is employed [12-15, 21, 23, 36, 37, 41] to numerically solve for the discrete approximation of Eq. (1) with CEM boundary conditions. The domain $(\Omega)$ is first divided into a finite number of small triangular elements as illustrated in Figure 2 (c) with $N_{n}$ nodes where a constant conductivity is assumed within each element. The weak solution $\left(u_{F E M}\right)$ to the problem governed by Eq. (1) is then estimated by taking the sum of the nodal voltages of each $i$ th element multiplied by each of its corresponding piecewise linear basis functions $\left(\varphi_{i}\right)$ as:

$$
u \approx u_{F E M}=\sum_{i=1}^{N_{n}} u_{i} \varphi_{i}
$$




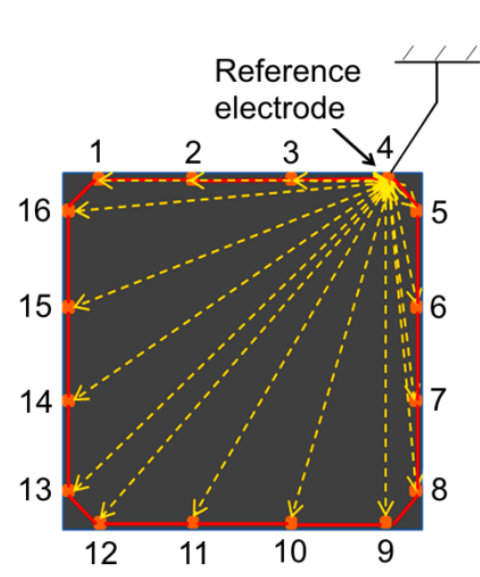

(a)

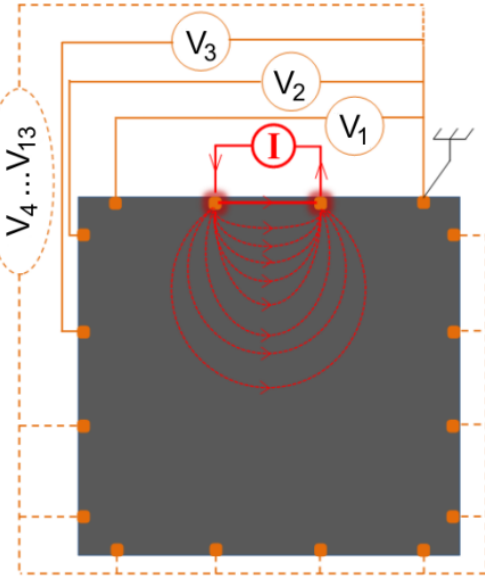

(b)

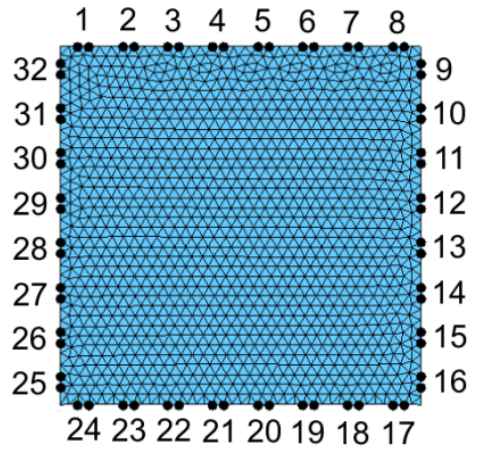

(c)

Figure 2. Illustration of EIT methodology adapted for our CNT composite sensor: (a) current injection route (solid red line) along the boundary and voltage measurement protocol (dashed yellow lines) for a 16-electrode (boundary dots) sensor, (b) detailed illustration of the series of voltage measurements (i.e. $\mathrm{V}_{1}$ to $\mathrm{V}_{13}$ ) corresponding to a selected current injection electrode pair (for simplicity, only 16 electrodes are shown in (a) and (b)), and (c) FE mesh with 2336 triangular elements used in modeling of our actual 32-electrode CNT composite sensor.

Details about the FE model used in this study are further explained in Section 2.3. For a finite set of injecting current, the FE system for the forward problem is commonly formulated as a system of linear equations $[37,41]$ :

$$
\left[A_{M}+A_{Z} A_{W} A_{W}^{T} A_{D}\right][u U]=[0 I]
$$

where

$$
\begin{gathered}
{\left[A_{M}\right]_{i j}=\int_{\Omega}^{1} \sigma \nabla \varphi_{i} \cdot \nabla \varphi_{j} d x d y} \\
{\left[A_{Z}\right]_{i j}=\sum_{l=1}^{L} \frac{1}{z_{l}} \int_{e_{l}}^{1} \varphi_{i} \varphi_{j} d S} \\
{\left[A_{W}\right]_{i}=-\frac{1}{z_{l}} \int_{e_{l}}^{1} \varphi_{i} d S} \\
{\left[A_{D}\right]=\operatorname{diag}\left(\frac{\left|e_{l}\right|}{z_{l}}\right)}
\end{gathered}
$$


with $i, j=1,2, \ldots, N_{n}, l=1,2, \ldots, L,\left|e_{l}\right|$ is the length of the electrode $e_{l}$ (in 2-D), and $z_{l}$ is the contact impedance between the electrode and the sensing medium. In this algebraic system, $\left[A_{M}\right]$ represents the usual system matrix for the governing equation to the numerally meshed 2-D domain while $\left[A_{W}\right],\left[A_{D}\right]$ and $\left[A_{Z}\right]$ impose the CEM boundary conditions $[37,41]$. The unknown nodal voltages $[u]$ and boundary voltages $[U]$ at electrodes are then solved with the known injecting current $[I]$ at the electrodes. These calculated voltages are used to construct the Jacobian matrix in the inverse problem as explained in the following section.

\subsection{Inverse Problem}

The inverse problem reconstructs the sensor's internal distribution of conductivity change in accordance with the voltage measurements at all electrodes resulting from the adjacent current injection scheme (Figure 2 (a) and (b)). This represents an ill-posed non-linear problem and the result is sensitive to modeling errors and measurement noise [36,37,41]. In this study, for solving the inverse problem we used a maximum a posteriori (MAP) reconstruction approach which was developed by Adler and Guardo [42]. This MAP algorithm makes the Gaussian assumptions for the a posteriori distribution and is a one-step linearization solver reconstructing the normalized change in electrical conductivity between the intact and damaged condition of the sensor. This probabilistic method yields the estimate $\left(\left[\Delta \sigma / \sigma_{0}\right]\right)$ following the regularized inverse as $[14,15$, 42]:

$$
\left\{\frac{\Delta \sigma}{\sigma_{0}}\right\}=\left[\left(J^{T} W J+\lambda R\right)^{-1} J^{T} W\right]\left\{\frac{\Delta U}{U_{0}}\right\}=B\left\{\frac{\Delta U}{U_{0}}\right\},
$$

where

$$
\left.[J]_{i j=-} \sum_{i=1}^{2}(\nabla u)_{i}(\nabla u)_{j}\right)_{j} x d y(\quad \text { for isotropic 2-D medium [36] })
$$

in which, $[W]$ is the covariance matrix containing the inverse variance of noise for every voltage measurement and then represents the noise level of the obtained voltage measurements; $[R]$ is the regularization matrix imposing conditions of smoothing and stabilization by using a spatially invariant Gaussian high-pass filter to treat the measurement noise; $\lambda$ is the regularization parameter controlling the amplification of noise in the reconstructed images $[14,15,42]$. $[B]$ is the image reconstruction matrix corresponding to a given $\lambda ;\left[U_{0}\right]$ represents baseline 
measurements from the undamaged state of the sensor; $[U]$ includes the measurements taken after damage may have occurred; $[\Delta U]=[U]-\left[U_{0}\right]$; Jacobian matrix $[J]$ relates the changes in the measured voltages at the electrodes to the changes in the conductivities of the elements shown in Figure 2 (c).

In this study, $[W]$ is separately represented by a sparse and an identity matrix. For the sparse matrix, it is assumed that Gaussian noise exists. The identity matrix on the other hand assumes zero-mean Gaussian white noise, ignoring the physical errors within the specimen. The regularization parameter $(\lambda)$ is determined according to the resulted noise figure $(N F)$ of MAP algorithm, in which $N F$ is defined as the signal-to-noise-ratio $(S N R)$ of the voltage measurements $\left(S N R_{U}\right)$ divided by the SNR of the reconstructed conductivity $\left(S N R_{\sigma}\right)$ according to [42]:

$$
N F=\frac{S N R_{U}}{S N R_{\sigma}}=\frac{\left(1^{t} Z\right) \sqrt{N \cdot \operatorname{trace}\left(A B W^{-1} B^{t} A\right)}}{\left(1^{t} A B Z\right) \sqrt{M \cdot \operatorname{trace}\left(W^{-1}\right)}}
$$

where, $N, M$, and $[A]$ are the number of elements, number of voltage measurements and the diagonal matrix composing of the areas of the $i$ th element, respectively. $[Z]=\left([U]-\left[U_{0}\right]\right) /\left[U_{0}\right]$ and $[B]$ is defined in Eq. (8). In order not to under- or over-smooth the reconstruction, an optimal regularization parameter $\left(\lambda_{\text {optimal }}\right)$ was determined when the reconstructed image results in $N F=1$ $[14,15,42]$.

\subsection{Numerical Modeling}

The finite element method is employed along with partial calculation routines selected from the electrical impedance tomography and diffuse optical tomography reconstruction software (EIDORS) $[41,43,44]$ through MATLAB (R2012a, MathWorks $\left.{ }^{\circledR}\right)$. The geometry and mesh generation of the FE models for our CNT-based composite sensors were developed using a MATLAB-based mesh generator, DistMesh [45]. The final mesh of the FE models consists of 1238 nodes and 2336 2-D triangular elements as shown in Figure 2 (c) where each electrode was modeled as two adjoining nodes represented by black dots. By applying the MAP algorithm, the difference in voltage measurements between the undamaged and damaged states is immediately calculated and used for the EIT reconstruction. In this way, the experimental errors associated with the contact impedance, poor electrode contact and electrode mismatches do not change in the EIT measurements for both states, then by subtracting the initial measurements we avoid 
these errors influencing the reconstruction of the damaged specimens [19, 42]. Here, the complete electrode model (CEM, [46]) was employed and a unit contact impedance value was assumed for all 32 electrodes. For the forward problem, the initial electrical conductivity of the FE model was considered isotropic. The final EIT reconstruction map for each specimen was achieved in correspondence to $\lambda_{\text {optimal }}$ (defined in Section 2.2). Specifically, MATLAB was used to handle the EIT calculations based on a customized script to perform the direct inversion of the system matrix. This large sparse matrix is in the size of 1270 by 1270 (with conditioning numbers of $3 \sim 6 \times 10^{17}$ for our FEMs) and stored in the MATLAB program. The solution to the forward problem takes about 4 minutes of computational time on a $2.40 \mathrm{GHz}$ desktop computer with 8 GB RAM and 2-3 minutes of computational time to solve the inverse problem.

\section{Experiment}

\subsection{Sensor Fabrication Process}

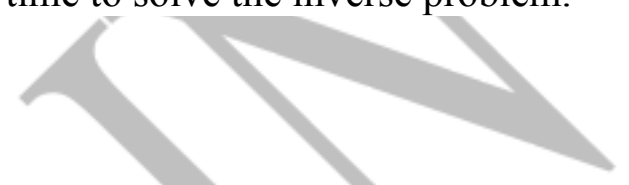

A sizing approach was used to fabricate the CNT-based composite sensor by employing a nonwoven aramid veil (34 g/m², Technical Fiber Products Inc.) as the non-conductive carrier fabric. This $0.5 \mathrm{~mm}$ thick fabric consists of $12.7 \mathrm{~mm}$ long randomly oriented short fibers, as shown in Figure 3 (a). The trimmed fabric was dipped into a commercially-available CNT sizing agent (SIZICYL ${ }^{\mathrm{TM}}$ XC R2G, Nanocyl), as shown in Figure 3 (b), which had been diluted with distilled water (1:2 by weight) and sonicated for 15 minutes using a bath sonicator (Branson ${ }^{\circledR}$ 1510, Branson Ultrasonics Corp.). The CNT-modified fabric was then dried at $130^{\circ} \mathrm{C}$ and infused with epoxy resin to form the final CNT-based composite sensor. After finishing the resin infusion, the composite sensor was cured in the oven at $130^{\circ} \mathrm{C}$ for 6 hours. For a detailed discussion of the sizing approach, the reader is referred to our previous studies [9,24]. The resulting final CNT-based composite sensor has a uniform thickness of $0.47 \mathrm{~mm}$ across the entire area with 0.75 wt. $\%$ CNT. Through this process, each individual short fiber becomes conductive and the whole nonwoven fabric turns into a dense network of randomly connected resistors. Figure 3 (c) illustrates the sensor's flexibility and the final product. As discussed in our previous work [9], this CNT-sizing-based two-step technique employs a dip-coating process using a prepared low CNT concentration aqueous solution which significantly reduce the material and 
labor cost comparing with other studies [3, 5, 18]. Specifically, solely coating the nonwoven fabric significantly improves the efficiency of CNTs as forming conductive networks within the nanocomposites by concentrating the limited amount of CNTs onto a small amount of fibers. Therefore, our approach is low cost, simple to setup and operate, and able to be easily scaled up.

In our prior research scanning electron microscopy (SEM) was utilized to study the morphology of the CNT coating on the aramid nonwoven fabric. The CNT layer uniformly coats the individual fibers, indicating good wettability of the sizing [9]. The CNTs form a random conductive network that is supported by the aramid fibers.

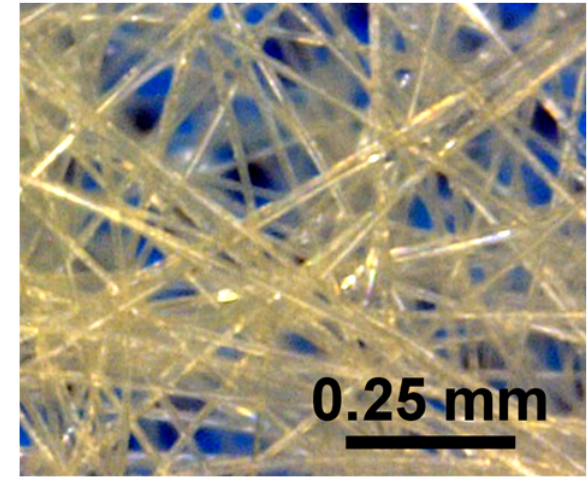

(a)

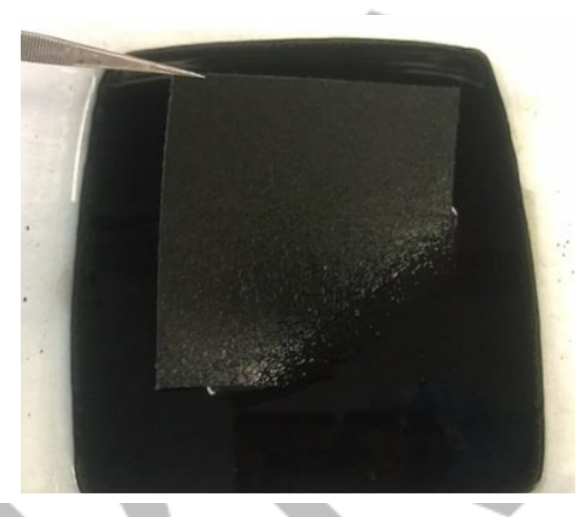

(b)

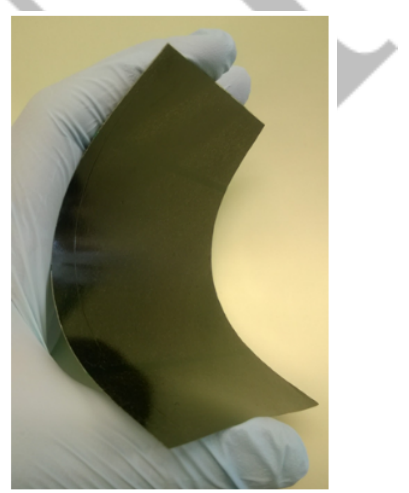

(c)

Figure 3. (a) Optical micrograph of nonwoven aramid fabric and its random fiber architecture, (b) coating of CNTs on fabric using the water-based CNT sizing (c) photo showing the flexibility of final CNT composite sensor.

\subsection{Preparation of Test Specimens}

Boundary electrodes were applied to the sensor in order to conduct the EIT measurements (described in Section 2.1). A 32-electrode arrangement with eight electrodes equally spaced along each boundary was selected for this study, as shown in Figure 4 (a). For each electrode location, an area of $3.2 \mathrm{~mm} \times 3.2 \mathrm{~mm}$ was coated with a conductive silver paint (SPI Supplies ${ }^{\circledR}$, Structure Probe, Inc.). Two lead wires were then attached to each electrode using a conductive epoxy (EPOXIES ${ }^{\circledR}$ 40-3900, Epoxies, Etc.). According to the manufacturer the electrical resistivity of the conductive epoxy is $0.0001 \Omega-\mathrm{cm}$. The conductive epoxy was cured at $90^{\circ} \mathrm{C}$ for 30 minutes. Finally, the sensor was attached to a $178 \mathrm{~mm} \times 114 \mathrm{~mm} \times 3.2 \mathrm{~mm}$ glass fiber 
composite (G-10/FR4, Professional Plastics, Inc.) using a 5-minute epoxy (Loctite ${ }^{\circledR}$ E-00CL Hysol ${ }^{\circledR}$, Henkel) to form the final test specimen. The non-conductive composite has an elastic modulus of $18.6 \mathrm{GPa}$ and flexural strength of $379 \mathrm{MPa}$, as reported by the manufacturer. A total of three test specimens were prepared for this study with reference conductivities for Specimen 1, 2, and 3 of 3.0, 3.5 and, $3.4 \mathrm{~S} / \mathrm{m}$, respectively. Figure 4 (b) shows an example of the three specimens.

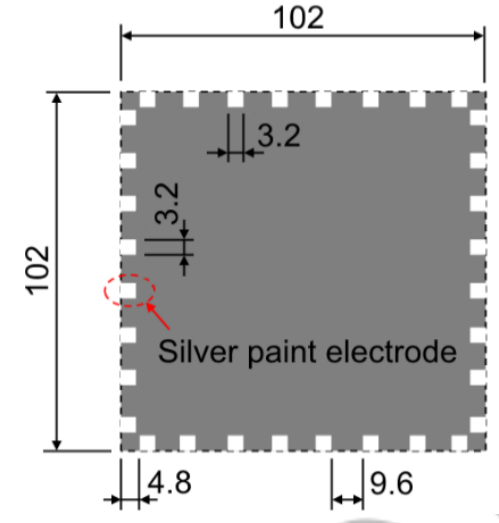

(a)

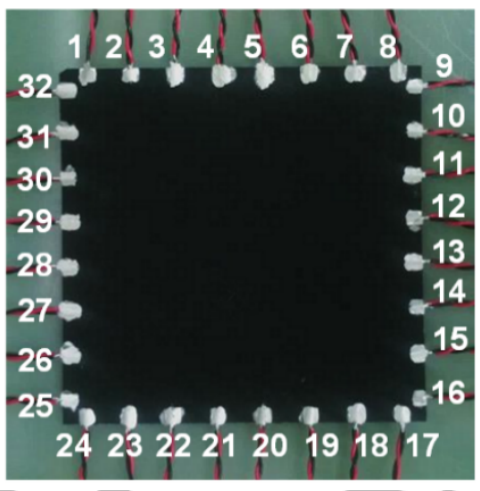

(b)

Figure 4. (a) Illustration of the electrode array for a 32-electrode CNT composite sensor made and (b) photograph showing the final EIT specimen used in this study where CNT composite sensor is attached on a nonconductive composite. Dimensions in ( $\mathrm{mm})$.

\subsubsection{Specimen 1: Square Holes}

Three identical holes each with a size of $12.7 \mathrm{~mm} \times 12.7 \mathrm{~mm}$ were introduced to Specimen 1 by successively removing the sensing area at three random locations. These holes introduce an infinite resistance increase in the electrical field at those locations and correspond to $1.6 \%, 3.1 \%$, and $4.7 \%$ of the total sensing area. This test aimed at validating the feasibility and sensitivity of our methodology to localized damage occurring at different locations simultaneously.

\subsubsection{Specimen 2: Simulated Crack}

A $25.4 \mathrm{~mm}$ long narrow notch was cut using a razor-blade on Specimen 2 to introduce a local discontinuity in the sensor, simulating a crack. Figure 9(a) shows the sensor with a $0.4 \mathrm{~mm}$ 
wide cut (i.e., aspect ratio $=64$ ). This localized damage represents $0.1 \%$ of the total sensor area. The goal of this test was to evaluate the sensitivity of our methodology to detect damage with a large aspect ratio, similar to an actual crack.

\subsubsection{Specimen 3: Impact Damage}

Impact damage is a common problem for composites because of their relatively low out-of-plane strength [47]. The objective of this specimen is to examine the sensitivity of the methodology to the damage severity. After specifying an impact energy level of $6.7 \mathrm{~J} / \mathrm{mm}$ in accordance with ASTM-D7136 [48], Specimen 3 was impacted multiple times with 21-J nominal energy impacts using an Instron Dynatup 9200 drop weight tester with a $12.7 \mathrm{~mm}$ blunt hemispherical tup hitting the center of this specimen. Figure 5 shows the impact test setup where the specimen was clamped on along its long edge. The specimen was impacted six times. After the sixth impact the tup completely perforated the composite laminate. Snapshots of the impacted specimen are shown and discussed further in Section 4.4.

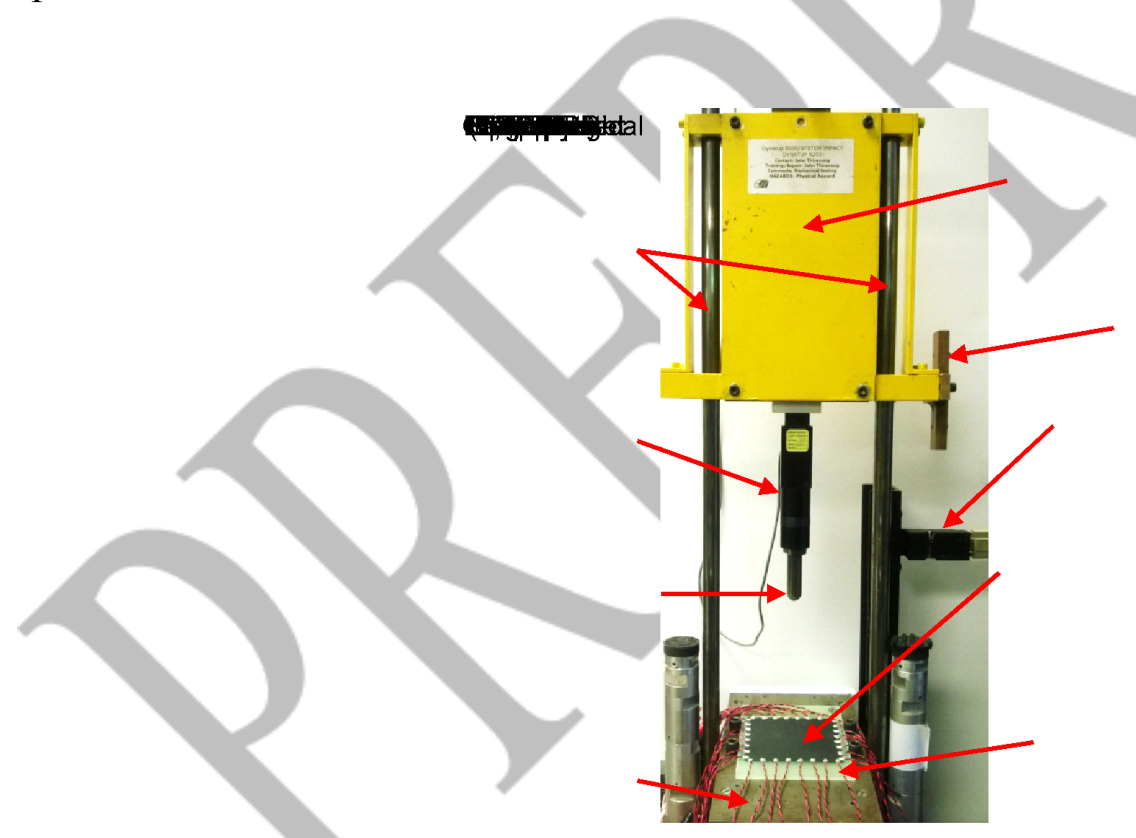

Figure 5. Photo of impact test apparatus with Specimen 3. 


\subsection{Non-Destructive Characterization}

For each specimen, visual inspection, EIT measurements, and infrared (IR) thermography were performed after each impact. Prior to the initial impact boundary voltage measurements were obtained and used as the undamaged reference measurement.

\subsubsection{EIT Measurements}

For EIT measurements current was sourced using the adjacent (neighboring) pattern [34]. Compared to the traditional adjacent current-voltage schemes, we measured the voltage differences from all other remaining pairs of electrodes with respect to a reference electrode as illustrated in Figures 2(a) and 2(b) to increase the number of independent measurements and the overall sensitivity at the center of the sensor. The domain used in the EIT inversion has exactly the same dimensions at the specimen. As highlighted in Figure 2(a) the simplified samples are 2-D square sensors with 16 electrodes, where all 16 current injection pairs are located successively along the boundary (i.e., clockwise from the first pair of electrodes \#1 and \#2, the second pair of electrodes $\# 2$ and $\# 3 . .$. , to the $16^{\text {th }}$ pair of electrodes $\# 16$ and $\# 1$ ) and the resulting voltage differences are measured from electrode \#1 to \#16 between the grounded reference electrode \#4 shown as the yellow-dashed lines. To minimize errors caused by the contact impedance at current-carrying electrodes, only the voltage measurements taken from the pairs of electrodes not overlapping the current injecting electrodes are used in the EIT reconstruction algorithm. For example, when the current is applied between electrodes \#2 and \#3, as shown in Figure 2 (b), 13 voltage measurements are acquired and saved. In this way, a total of $13 \times 14=$ 182 voltage measurements are obtained. Correspondingly, a complete measurement set of $29 \times$ $30=870$ differential voltages are acquired for our CNT-based composite sensor with 32 electrodes (shown in Figure 4 (b)). Following the commonly accepted minimal contrast of the EIT approach defined in accordance with the number $\left(N_{\text {ivol }}\right)$ of independent differential voltage measurements as [49]:

$$
\text { Nominal resolution }=\frac{1}{\sqrt{N_{\text {ivol }}}} \times 100 \%
$$

Our EIT methodology is believed to hold the minimal planar contrast of $1 / \sqrt{870}=3.4 \%$. 
The data acquisition system used to perform the current-voltage measurements consists of three components: a current source meter, a voltmeter, and an electrical multiplexer. These were integrated and controlled by a customized National Instruments LabVIEW program. All 64 connection wires from the test specimens (shown in Figure 4(b)) were connected into a Keithley 3750-ST terminal block attached to a Keithley 3706A multiplexer. By following the prescribed current injection pattern, a DC current was applied using a Keithley 6430 source meter via an electrode pair consisting of a current source and current sink. In order to avoid Joule heating while providing an accurate measurement with minimal noise, $10 \mathrm{~mA}$ was selected for all EIT experiments in accordance with the conductivity of the fabricated sensor. The resulting voltage measurements were collected using a Keithley 2182A nano-voltmeter. Electrode \#8 per Figure 4 (b) was assigned as the fixed ground electrode. A full set of EIT data was obtained until the current as injected to all pairs of adjacent electrodes excluding the two pairs involving the ground electrode. The initial (reference) conductivity of the specimens was determined in accordance with the average resistance measured in its horizontal (i.e., electrodes of \#32 and \#9, electrodes \#31 and \#10..., electrodes \#25 and \#16) and vertical directions (i.e., electrodes of \#1 and $\# 24$, electrodes of $\# 2$ and $\# 23 \ldots$, electrodes $\# 8$ and \#17). The conductivity of each sensor was calculated using the following equation:

$$
\sigma=\frac{1}{\rho}=\left(R \frac{A}{L}\right)^{-1}
$$

where $\sigma$ is the electrical conductivity, $\rho$ is the resistivity, $R$ is the measured electrical resistance in one direction, $L$ is the length and $A$ is the cross-sectional area of the sensing skin.

\subsubsection{Infrared Thermography}

Infrared thermography (IRT) is a non-contact non-destructive evaluation (NDE) technique frequently used for the inspection of civil structures [50,51], electronics [52], machinery [53], and composites [54, 55]. IRT utilizes an infrared detector to capture the infrared radiation emitted by an object [56]. For this study, active IRT was used to perform the post-damage inspection with the handheld IR camera (i7, FLIR ${ }^{\circledR}$ Systems, Inc.). Active IRT uses a heat source to produce thermal contrast between the damage and damage-free regions in an object. The entire specimen was heated up to a temperature of $125^{\circ} \mathrm{C}$ in the oven to maintain a 
consistent thermal reference for all IRT images, followed by cooling outside the oven. Due to the different thermal conductivities between the composite, the adhesive layer, and the damaged and undamaged regions within the sensor, the heat energy dissipates from the specimen at different rates which results in temperature differentials in different areas. For each specimen, multiple thermograms were taken at different temperatures and the one with the best resolution of showing the damage is reported.

\section{Results and Discussion}

\subsection{Baseline Measurements}

Prior to damaging the specimen, EIT measurements were conducted to obtain the baseline reconstruction maps and quantify the background noise level. As detailed in Section 2.2, two kinds of covariance matrices including the sparse and identity matrix were employed in this study. Figure 6(a) shows a photo of the undamaged sensor used for the three damage cases. As displayed in Figure 6(b), the sparse covariance matrix leads to scattered background noise and corresponds to a $\lambda_{\text {optimal }}=4.1289 \times 10^{-7}$ (defined in Section 2.2 ). The identity covariance matrix produces concentrated background noise only along the boundary as presented in Figure 6(c) and the corresponding $\lambda_{\text {optimal }}=0.0519$. It can be seen that both approaches cause minimal background noise $(<-0.5 \%$ conductivity change $)$.

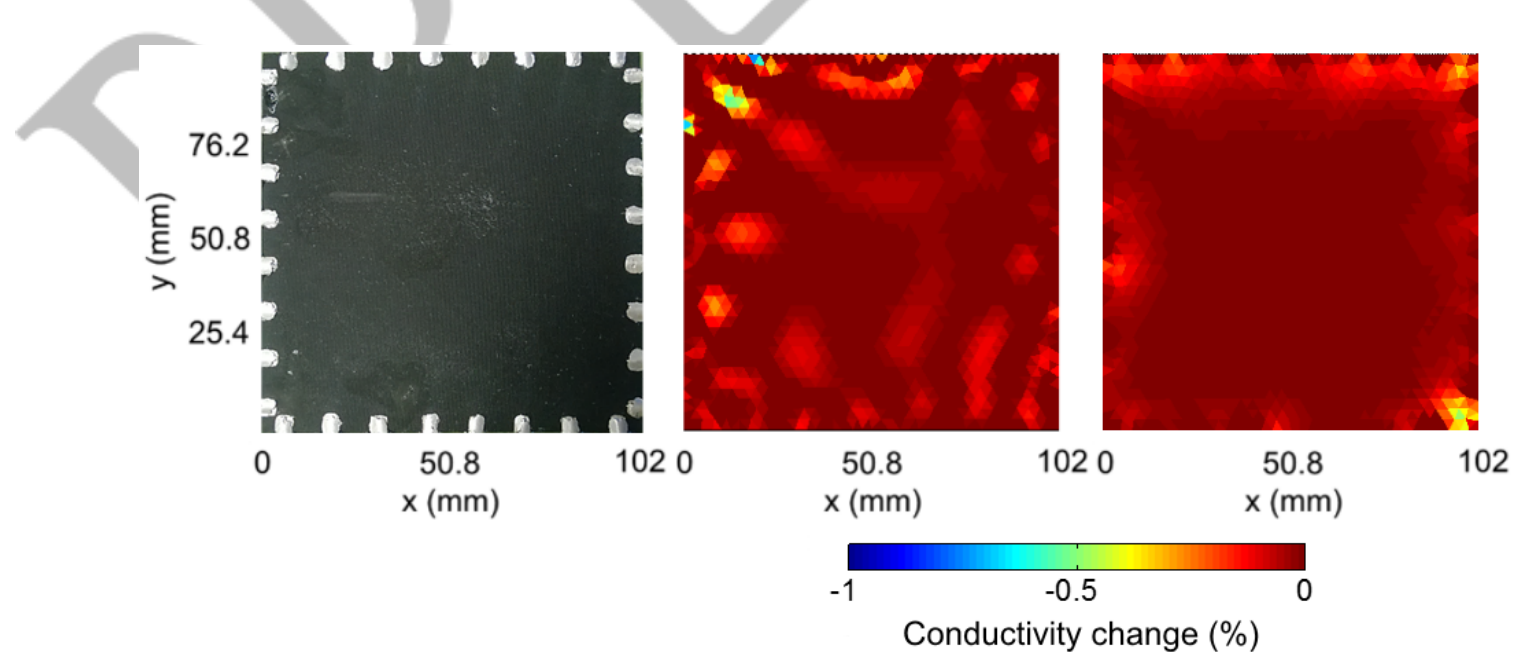

(a)

(b)

(c) 
Figure 6. Baseline reconstruction results: (a) photo of undamaged sensor, and EIT maps of normalized conductivity change for (b) Specimens 1 and 2 using sparse covariance matrix, and for (c) Specimen 3 using identity covariance matrix.

\subsection{Specimen 1: Square Holes}

The executed EIT reconstructions for Specimen 1 corresponding to the three-step damage test described in Section 3.2.1 were obtained by using a unit $N F$ with $\lambda_{\text {optimal }}=6.3939 \times 10^{-7}$ (per Section 2.2) and are shown in Figures 7(a2) through 7(c2). Photos of Specimen 1 for the different damage stages are shown in Figures 7(a1) through(c1). The dashed squares represent the holes removed from the CNT-based composite sensor. While our EIT methodology accurately maps the location of all three holes, the damage severity and shape are slightly overestimated and shown as round-shaped areas with a negative conductivity change. A possible reason may be that the sensor is treated as an electrically homogeneous object where, in reality, it likely contains some local anisotropy due to the fibrous structure. Quantitatively, the predicted damage areas are $2.87,5.91$, and $10.3 \%$ of the total sensing area, which results in an 84,89 , and $119 \%$ overestimation compared to the actual damage size, respectively.

As damage is added to the sensor more background noise is evident in the EIT maps. Figure 7(c3) shows an IRT temperature map for Specimen 1 for the case where all three square holes are present. The shape of the holes is distinctly represented by the darker (hot) lines indicating non-uniform heat flux occurring along the sharp edges of the discontinuities. The electrodes are also clearly observed as yellow-green dots corresponding to a much lower temperature because the thermal conductivity of the silver-filled epoxy electrodes is much higher than the rest of the specimen. In short, we were able to demonstrate that it is feasible to detect and map damage at multiple locations simultaneously using our EIT methodology. 
(1)

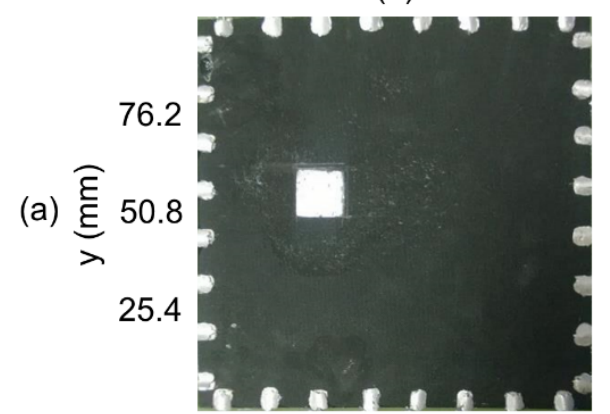

(b)
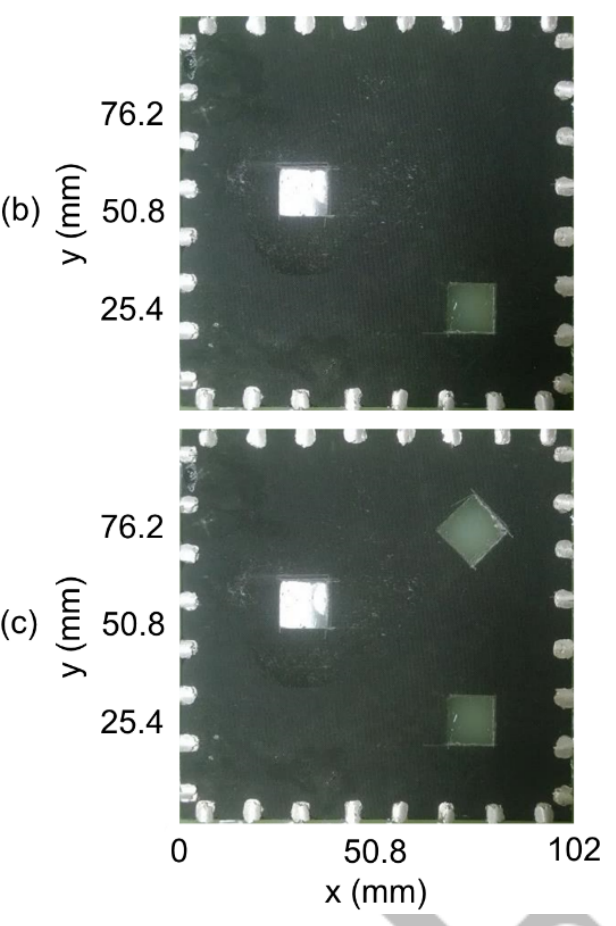

(2)
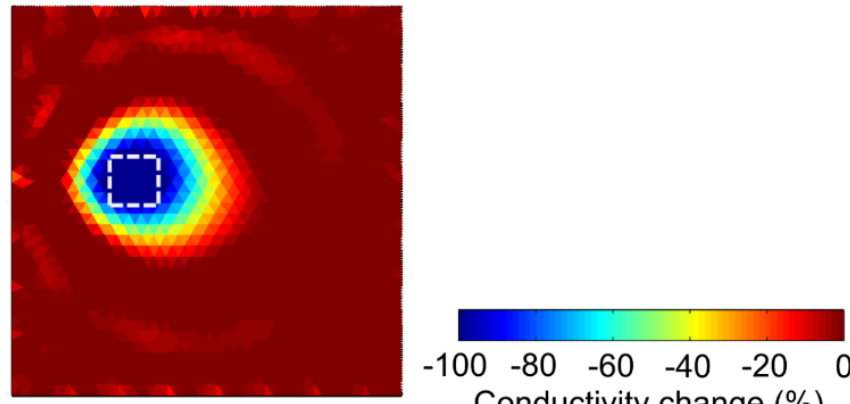

Conductivity change (\%)
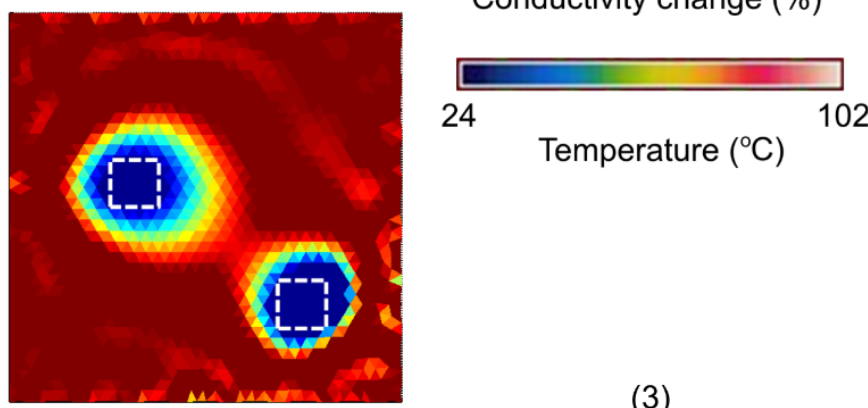

(3)

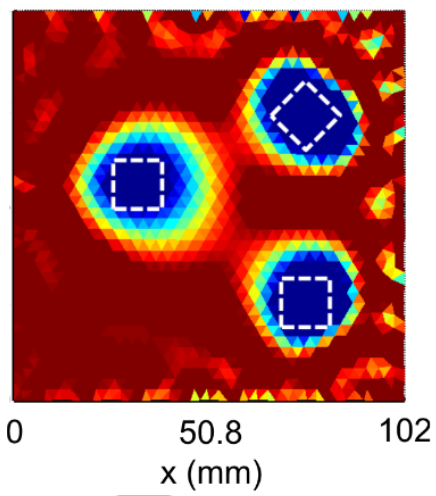

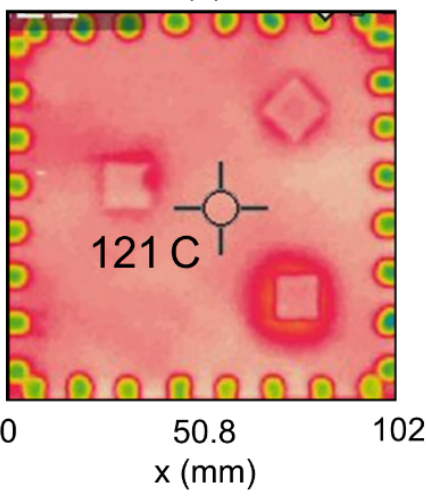

Figure 7. Experimental results: Columns show (1) Photo of CNT composite sensor on Specimen 1, (2) EIT maps of normalized conductivity change, and (3c) temperature map from IR thermography. Rows (a) through (c) correspond to the number of square holes.

A histogram of the elementary results from the EIT reconstruction is shown in Figure 8. It can be observed that the number of elements with $\geq-100 \%$ change in conductivity are 67,138 and 240 corresponding to one, two and three square holes, respectively. At the same time, the number of elements with no conductivity change decreases. Both trends follow a linear fashion, which validates the use of the linear reconstruction algorithm of MAP. These observations are in line with the experimental study conducted by Loyola et al. [14, 15]. 


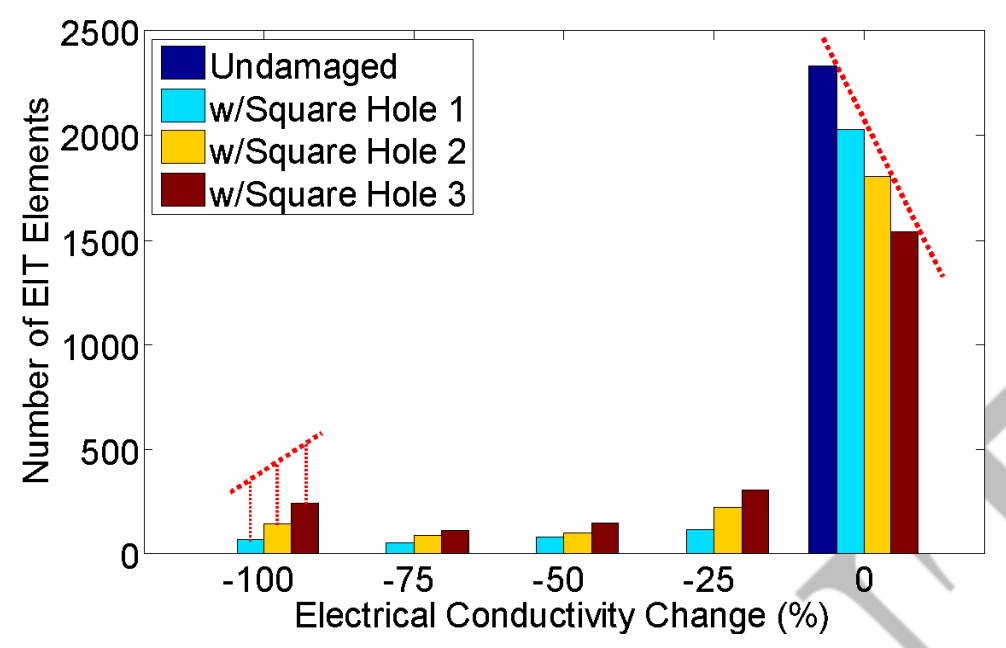

Figure 8. Distribution of the obtained 2336 EIT element results from each damage case.

\subsection{Specimen 2: Simulated Crack}

A photo of Specimen 2 is shown in Figure 9(a), and the obtained EIT map of Specimen 2, as described in Section 3.2.2, is shown in Figure 9(b). Similar to the previous case, the reconstruction was executed using the sparse covariance matrix when solving the inverse problem. The $\lambda_{\text {optimal }}$ corresponding to a unit $N F$ (described in Section 2.2) was found to be $2.0842 \times 10^{-7}$. As a result, these maps of normalized conductivity change for Specimens 1 and 2 show similar levels of noise and types of artifacts. It can be observed that the area with $\geq-100 \%$ conductivity change fully covers the simulated crack. In addition, the EIT reconstructed area with localized conductivity change is stretched along the diagonal direction. Figure 9(c) shows an IRT thermogram for Specimen 2. The shape of the simulated crack is shown by a darker (hot) area indicating the non-uniform heat flux caused by the discontinuity. The electrodes are also visible, similar to Specimen 1. Both EIT and IRT images are able to locate the simulated crack. Although the exact shape is not resolved there is a clear aspect ratio to the damaged area, indicating an elongated area of damage. Nevertheless, we were able to demonstrate that it is feasible to detect and map a discontinuity with a large aspect ratio (64 in this case) using our EIT methodology. 
(a)

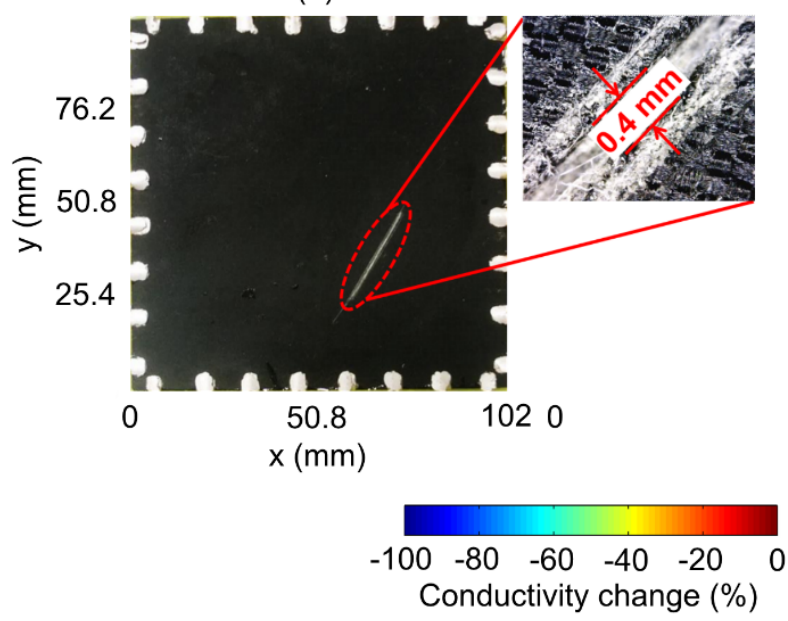

(b)
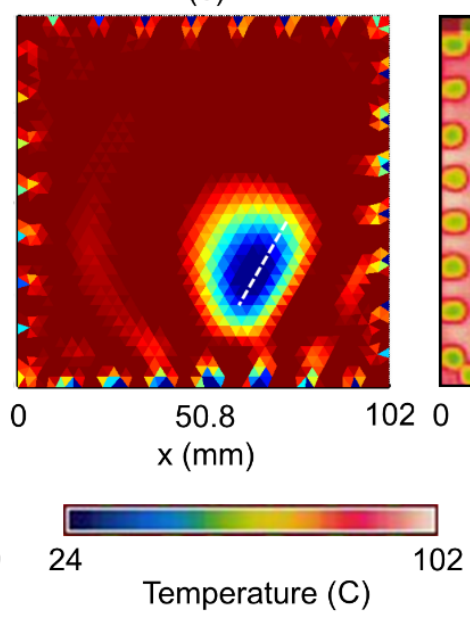

(c)

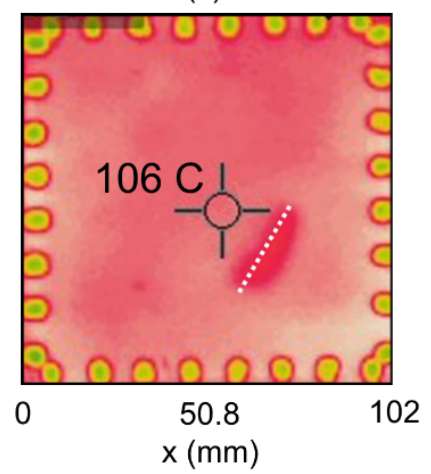

02

Figure 9. Experimental results: (a) Photo of CNT composite sensor on Specimen 2 with insert of artificial crack, (b) EIT maps of normalized conductivity change, and (c) temperature map from IR thermography.

A histogram of elementary results from the EIT reconstruction is plotted in Figure 10, in which $82 \%$ of the elements maintain unchanged in conductivity and $2.7 \%$ of them represent $\geq 100 \%$ decrease in conduction due to the applied damage on the sensing skin. This is consistent with the minimal planar contrast of $3.4 \%$ defined Section 3.3.1.

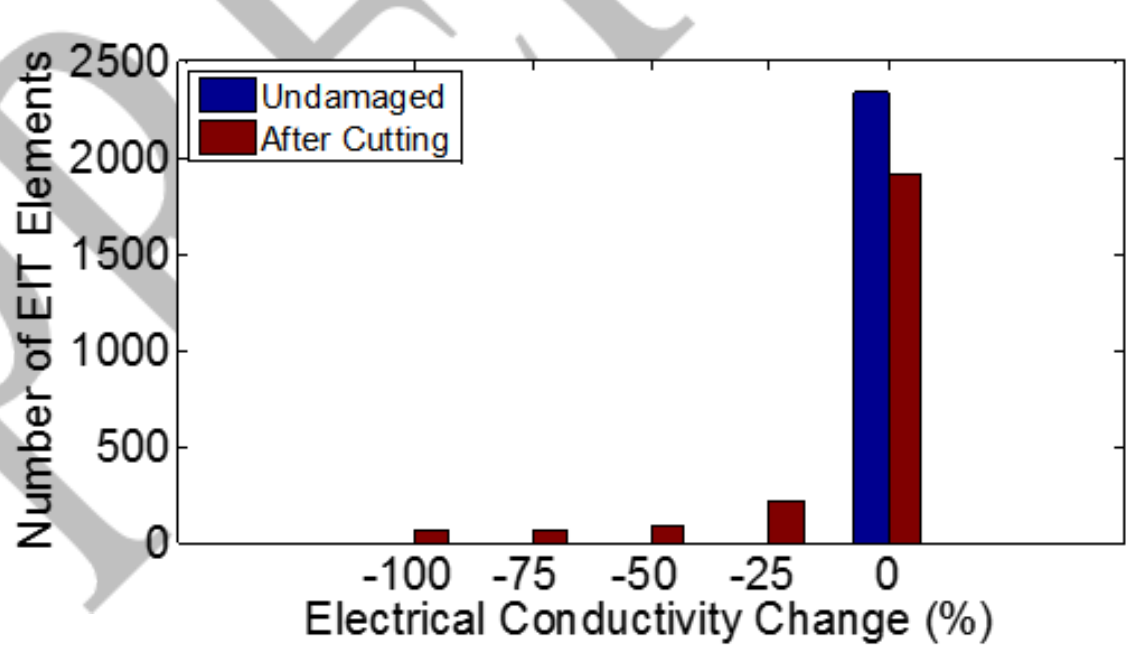

Figure 10. Distribution of the obtained 2336 EIT element results from each damage case. 


\subsection{Specimen 3: Impact Damage}

For Specimen 3 the identity matrix was used for $[W]$ to further reduce background noise. This implies that all boundary voltage measurements have uncorrelated noise (i.e., equal noise). Figures 11(a3) through 11(f3) present the resolved EIT maps of Specimen 3, which show minimized boundary noise and a clean background. The $\lambda_{\text {optimal }}$ corresponding to the unit $N F$ (described in Section 2.2) is found to be 0.0464 , which is much bigger than that for the previous two specimens. Similar to the previous two cases, the location of the impact damage is accurately predicted, although the size is slightly overestimated. Nevertheless, the EIT methodology is able to capture the evolution of the accumulated damage. The color gradient of the EIT identified damage areas is consistent with the severity of the imposed damage. After each impact, the specimen was visually inspected. Photos of the impacted top face (sensor) and the back face (composite laminate) taken during the six-step impact test are shown in Figures 11(a1) through 11(f1) and Figures 11(a2) through 11(f2), respectively. It is evident that damage in Specimen 3 accumulates with each impact. A description of the visual observations from the test is presented in Table 1 .

Table 1. Visual observations from the six-step 21-J impact test.

\begin{tabular}{|c|c|c|c|c|c|c|}
\hline \multirow{2}{*}{$\begin{array}{c}\text { Impact } \\
\text { no. }\end{array}$} & \multicolumn{3}{|c|}{$\begin{array}{l}\text { Impacted Face: Sensor } \\
\text { Figure } 11 \text { (a1) through (f1) }\end{array}$} & \multicolumn{3}{|c|}{$\begin{array}{l}\text { Back Face: Composite Laminate } \\
\text { Figure } 11 \text { (a2) through (f2) }\end{array}$} \\
\hline & Figure & Observations & $\begin{array}{c}\text { Damage } \\
\text { mode }\end{array}$ & Figure & Observations & Damage mode \\
\hline 1 & & $\begin{array}{l}3 \mathrm{~mm} \text {-diam. dot, } \\
\text { no sign of surface } \\
\text { cracking }\end{array}$ & $\begin{array}{c}\text { Barely } \\
\text { visible } \\
\text { damage }\end{array}$ & (a2) & $\begin{array}{l}10 \mathrm{~mm} \text {-square spot } \\
\text { with surface cracking }\end{array}$ & Matrix cracking \\
\hline & & $\begin{array}{c}7 \text { mm-diam. spot, } \\
\text { no sign of surface } \\
\text { cracking }\end{array}$ & $\begin{array}{l}\text { Slightly } \\
\text { visible } \\
\text { damage }\end{array}$ & (b2) & $\begin{array}{c}20 \text { mm- cross-shaped } \\
\text { spot with surface } \\
\text { cracking }\end{array}$ & Matrix cracking \\
\hline 3 & & $\begin{array}{c}\text { Barely visible } \\
\text { ring-shaped surface } \\
\text { cracking with } 10 \\
\text { mm-quatrefoil-shaped } \\
\text { central dent }\end{array}$ & $\begin{array}{l}\text { Minor } \\
\text { surface } \\
\text { cracking }\end{array}$ & (c2) & $\begin{array}{l}31 \text { mm-cross-shaped } \\
\text { spot with partial } \\
\text { bulging, surface } \\
\text { cracking }\end{array}$ & $\begin{array}{l}\text { Delamination / } \\
\text { Fiber fracture }\end{array}$ \\
\hline 4 & (d1) & $\begin{array}{c}22 \mathrm{~mm} \text {-diam. } \\
\text { ring-shaped cracking } \\
\text { with } 12 \\
\text { mm-quatrefoil-shaped } \\
\text { central dent }\end{array}$ & $\begin{array}{l}\text { Surface } \\
\text { cracking }\end{array}$ & (d2) & $\begin{array}{l}41 \text { mm-cross-shaped } \\
\text { bulge with broken } \\
\text { fiber at the corner }\end{array}$ & Fiber fracture \\
\hline 5 & (e1) & $\begin{array}{c}22 \text { mm-diam. ring } \\
\text { cracking with } 12 \\
\text { mm-quatrefoil-shaped } \\
\text { central dent and cross } \\
\text { cracking }\end{array}$ & $\begin{array}{l}\text { Sever } \\
\text { surface } \\
\text { cracking }\end{array}$ & (e2) & $\begin{array}{l}47 \mathrm{~mm} \text {-cross-shaped } \\
\text { bulge with broken } \\
\text { fiber along the edge }\end{array}$ & Fiber fracture \\
\hline
\end{tabular}




\begin{tabular}{|c|c|c|c|c|c|c|}
\hline 6 & (f1) & $\begin{array}{c}\text { hole penetrated through } \\
\text { the panel }\end{array}$ & Puncture & (f2) & $\begin{array}{c}41 \text { mm-cross-shaped } \\
\text { opening with broken } \\
\text { lamina }\end{array}$ & $\begin{array}{l}\text { Lamina fracture } \\
\text { and penetration }\end{array}$ \\
\hline
\end{tabular}

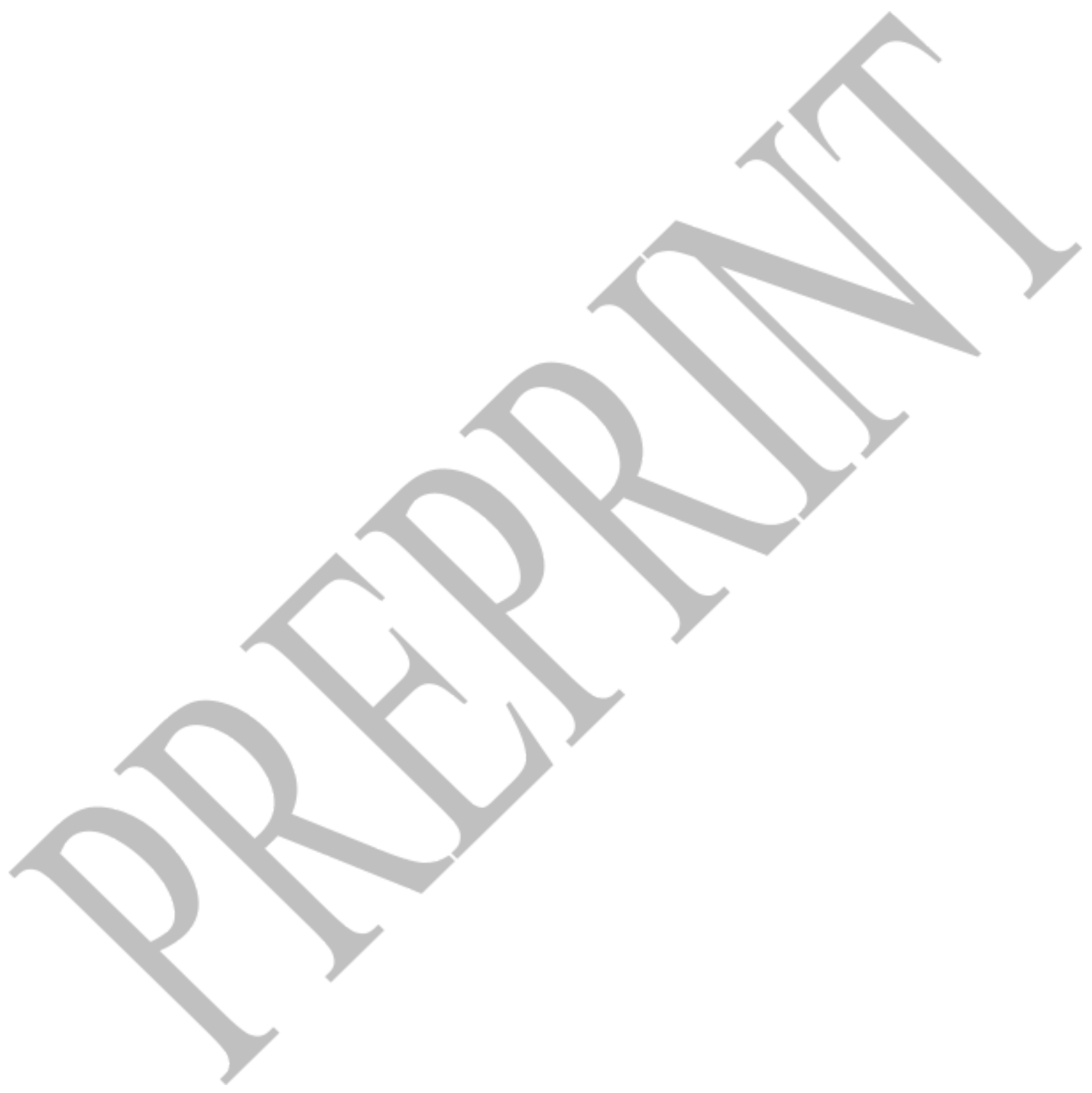


(1)

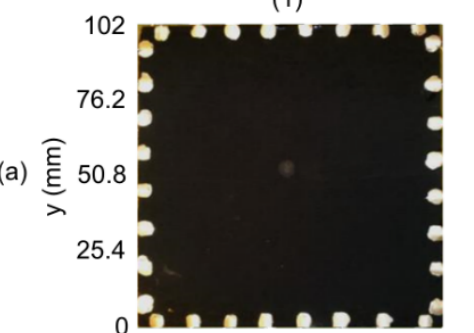

(b)

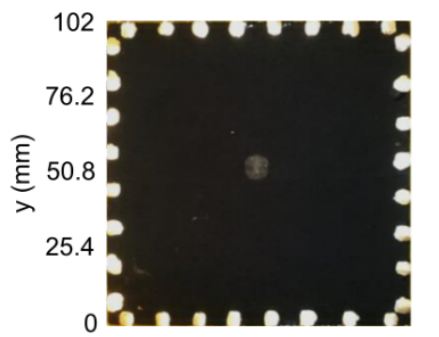

(c)
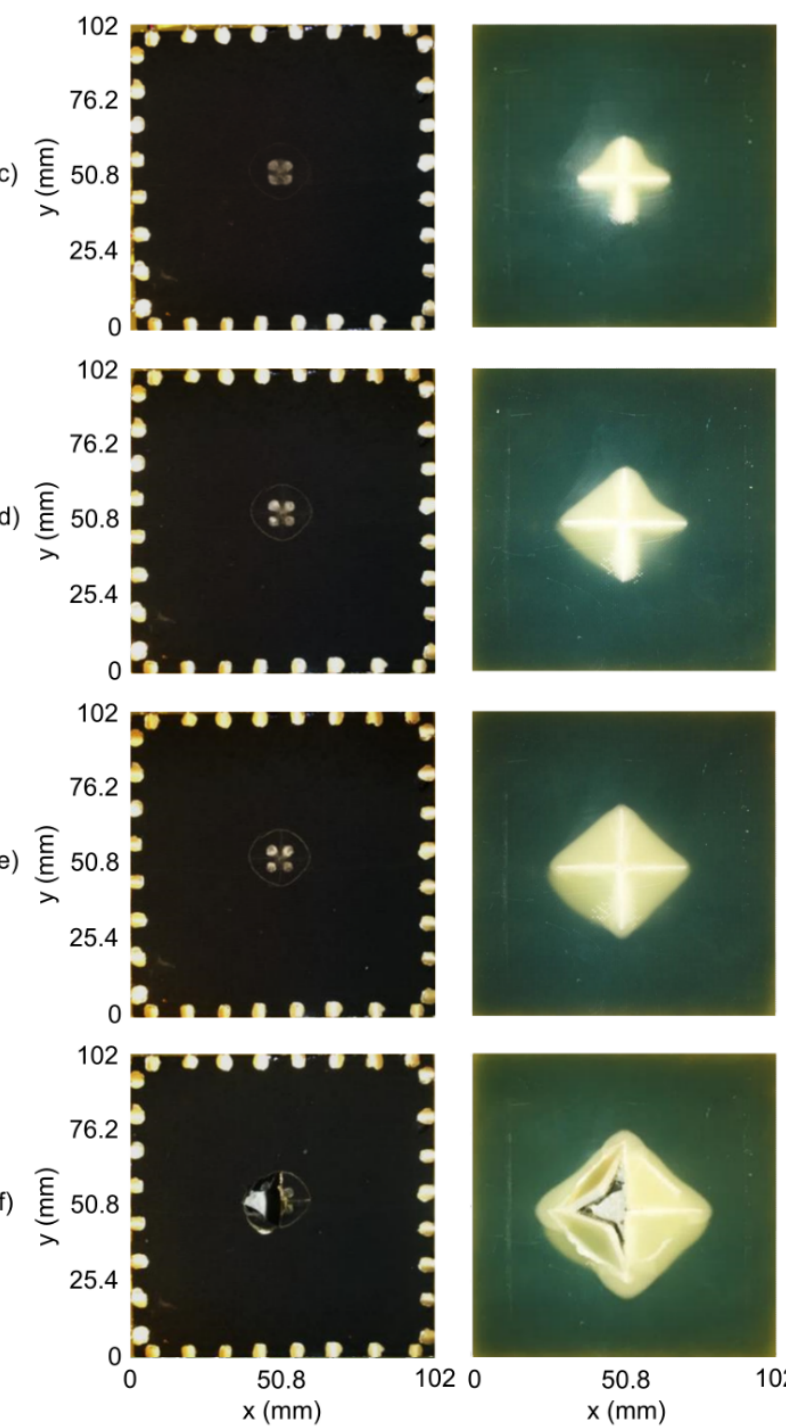
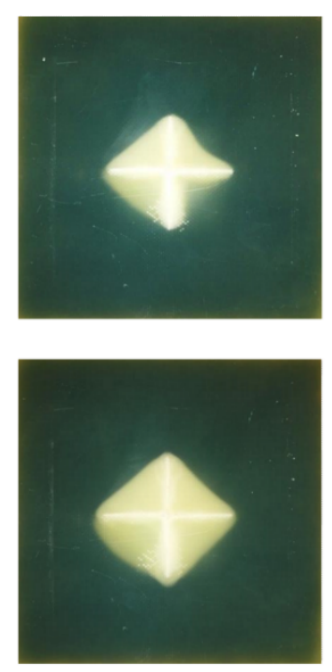

(2)
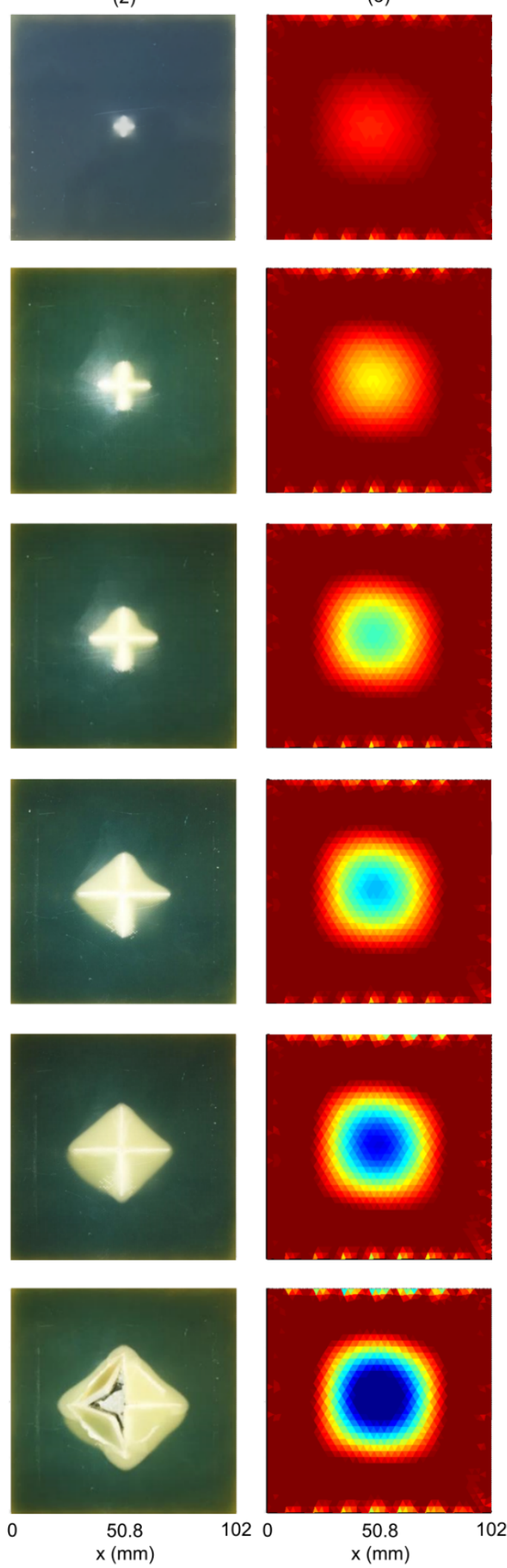

(3)
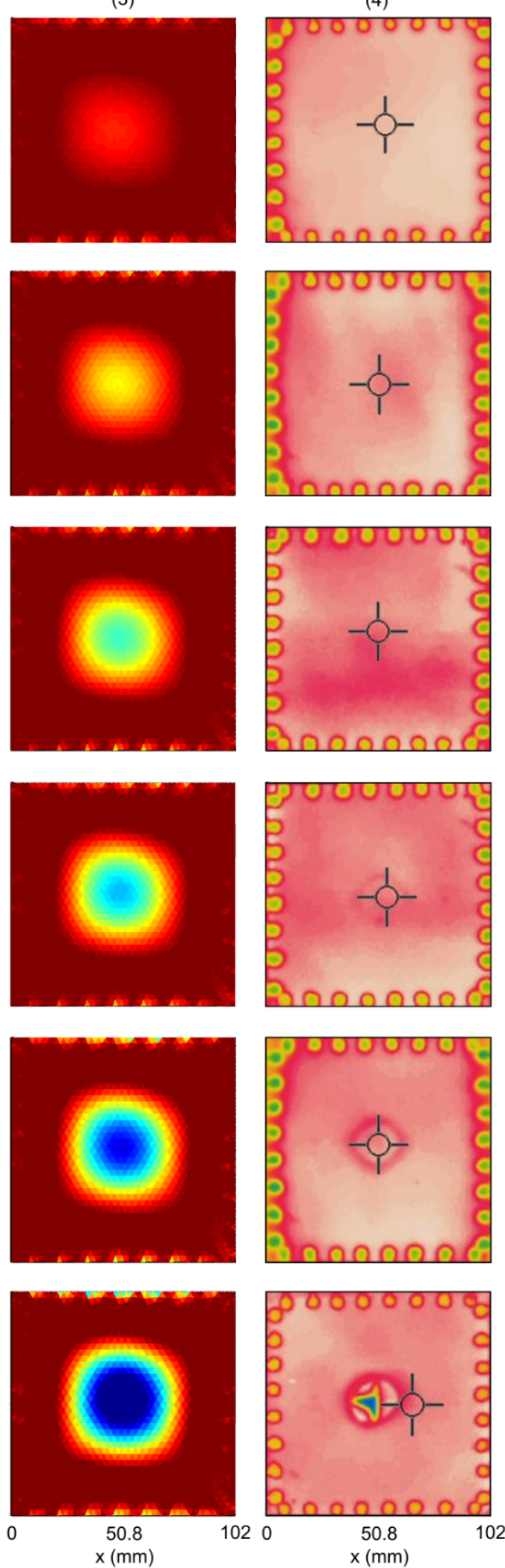
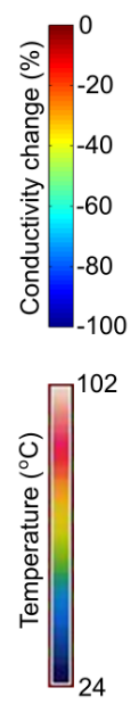

Figure 11. Experimental results: Columns show (1) Photo of CNT composite sensor on Specimen 3, (2) Photo of FRP panel (backside), (3) EIT reconstructions, and (4) thermograms from IR thermography. Rows (a) through (f) correspond to the number of 21-J impacts. 
The IRT temperature maps for Specimen 3 are shown in Figures 11(a4) through 11(f4). It can be observed that the temperature images for the first three impacts (Figures 11(a4) to 11(c4)) do not indicate any signs of impact damage. This is likely due to the fact that heat dissipation from the hot specimen to the cold ambient air can still be accomplished by the bridging fibers within the matrix-cracked zone. After the third impact, thermal images show the impact damage (Figures 11(d4) to 11(f4), and match the location of the boundaries of the real damaged areas on the test specimen. For this test, the EIT reconstructions were able to detect damage that was barely visible. IRT, on the other hand, required severe damage (i.e., surface cracking or fiber fracture) before the damage was detectable.

A histogram of the elementary conductivity changes for Specimen 3 is plotted in Figure 12. It can be observed that the number of elements with no conductivity change in the EIT maps correlate well to the six successive impacts in a bi-linear fashion, which is explained by visual observations included in Table 1: Since the first impact only causes minor damage in the specimen without permanently cracking the CNT composite sensor, the conductivity change is small at the impacted areas and a large number of conductive pathways around this area exist. Once the surface cracking is initiated after the 3rd impact, a large and direct reduction in local conductivity due to the damage on the CNT composite sensor is introduced. Next, the local change in conductivity continues declining with each successive impact and finally reaches the infinitesimal level due to the loss of sensing area after full penetration. From these results, we demonstrate that our damage detection methodology is capable of detecting, locating, and estimating the severity of accumulating damage due to impact.

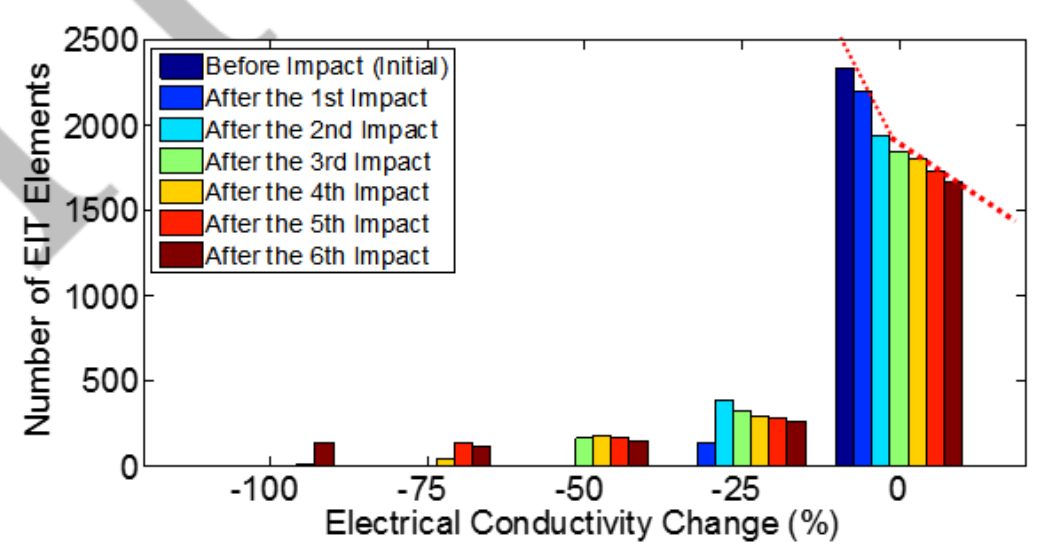


Figure 12. Distribution of the obtained 2336 EIT element results from each damage case.

\subsection{Discussion}

In general it can be observed that the resolved EIT maps are able to predict the location and size of damage. However, the shape is not predicted well and for the damage having a large aspect ratio, such as a crack, the area of damage is significantly overestimated. This relatively low spatial resolution is a common issue existing in all EIT applications [12-14, 16, 18, 19, 38] due to the inherent difficulties of this problem: (1) EIT is a diffusion problem where the injected electrical current propagates within the entire material $[37,41],(2)$ solving the severely ill-posed inverse problem continuously depends on the boundary voltage measurements, which are not always stable and accurately measurable [37], and (3) the smoothing regularization used in the inverse problem usually exaggerates the area with decreased conductivity [21]. It can also be seen from the EIT maps that artifacts appear at the electrodes and are rippling around the vicinity of conductivity-changed regions in Figures 7 and 9. Obviously, these artifacts are intensified as more conductivity discontinuities are introduced as for Specimen 1. Possible reasons for this could be the anisotropic conductivity around areas under the electrodes that violates the assumption of isotropic conductivity in the FE model and results in an inappropriate interpolation of the voltage measurements or the small electrode contact impedance leads the voltage measurements at the electrode depending on the neighboring mesh's conductivity in the FE model [57]. In addition, the use of a sparse $[W]$ (described in Section 2.2) in the MAP algorithm, assumes that the noise in the voltage measurements is correlated and causes relatively low measurement accuracy simulated in the inverse problem [34]. Meanwhile, the level of this correlated noise is amplified as more differential voltage measurements are associated with the enlarged regions with conductivity change (i.e., more holes on the sensor). Additionally, $\lambda_{\text {optimal }}$ (described in Section 2.2) values for Specimens 1 and 2 are very small, suggesting that these specific inverse problems are close to where linear approximation is valid. After employing an identity $[W]$ for Specimen 3, a comparatively large $\lambda_{\text {optimal }}$ is obtained. As discussed by others [12, 42], large $\lambda_{\text {optimal }}$ leads the regularization to amplify large singular value decomposition (SVD) components of the calculated voltage change corresponding to the conductivity change but to restrain the smaller SVD components. Since the large SVD components are associated with the 
voltage changes closer to the electrodes, therefore amplifying the large SVD components increases the contrast in the area around the electrode and results in the reduction of noise near the electrodes as shown in Figures 11(a3) through 11(f3). On the other hand, the small SVD component controls the reconstruction of conductivity at the interior of the object and to restrain them reduces the background noise and the resolution at the areas away from the electrodes in the EIT reconstruction map.

\section{Conclusions}

This research has established a methodology implementing a novel carbon nanotube-based composite sensor in conjunction with electrical impedance tomography (EIT) for detection and imaging of a variety types of damage. The novel sensor is based on a CNT-modified nonwoven aramid fabric and possesses isotropic electrical conductivity, mechanical robustness, and the ability to be adhered to complex surfaces. Additionally, the manufacturing process is cost efficient and allows the sensor to be scaled up for large engineering applications. A difference imaging-based EIT algorithm was implemented and adapted to enable 2-D spatial damage sensing capability of the sensor.

A series of tests were conducted to evaluate our methodology with a newly defined adjacent current-voltage measurement scheme. Damage included: (1) square holes cut into the sensor, (2) a narrow cut simulating a crack, and (3) progressive impacts on a composite laminate. The results demonstrate that it is possible to detect and locate damage as well as capture the severity of the accumulated damage. However, the size is typically overestimated and the shape not well represented. This is a particular problem for cracks, which have a large aspect ratio. Infrared thermography (IRT) images were also taken for comparison and produced comparable results. In some cases, however, our EIT methodology was able to detect the initiation of damage well before it was visible with IRT. Improvements on the EIT algorithm are planned in the future to increase the accuracy to predict the size and shape. Finally, the methodology will be scaled up and evaluated for large-scale structures in the laboratory. 


\section{Acknowledgements}

The support of this collaborative research effort by the National Science Foundation, CMMI Division, Award \# 1234830 (Dr. Kishor Mehta, Program Director) is greatly appreciated. The authors would like to thank Technical Fibre Products (TFP) for donating the nonwoven fabrics used in this research. We also would like to acknowledge Dr. Dirk Heider from Center for Composite Materials at University of Delaware for his supply of the infrared camera used in this research.

\section{References}

1. Thostenson, E.T.; Li, C.; Chou, T. Nanocomposites in Context. Composites Sci. Technol. 2005, 65, 491-516.

2. Pandey, G.; Thostenson, E.T. Carbon Nanotube-Based Multifunctional Polymer

Nanocomposites. Polymer Reviews 2012, 52, 355-416.

3. Hu, N.; Fukunaga, H.; Atobe, S.; Liu, Y.; Li, J. Piezoresistive Strain Sensors made from Carbon Nanotubes Based Polymer Nanocomposites. Sensors 2011, 11, 10691-10723.

4. Gao, L.; Thostenson, E.T.; Zhang, Z.; Chou, T. Coupled Carbon Nanotube Network and Acoustic Emission Monitoring for Sensing of Damage Development in Composites. Carbon 2009, 47, 1381-1388.

5. Kang, I.; Schulz, M.J.; Kim, J.H.; Shanov, V.; Shi, D. A Carbon Nanotube Strain Sensor for Structural Health Monitoring. Smart Mater. Struct. 2006, 15, 737.

6. Gao, L.; Thostenson, E.T.; Zhang, Z.; Chou, T. Sensing of Damage Mechanisms in Fiber-Reinforced Composites Under Cyclic Loading using Carbon Nanotubes. Advanced Functional Materials 2009, 19, 123-130.

7. Thostenson, E.T.; Chou, T. Carbon Nanotube-Based Health Monitoring of Mechanically Fastened Composite Joints. Composites Sci. Technol. 2008, 68, 2557-2561.

8. Lim, A.S.; Melrose, Z.R.; Thostenson, E.T.; Chou, T. Damage Sensing of Adhesively-Bonded Hybrid Composite/Steel Joints using Carbon Nanotubes. Composites Sci. Technol. 2011, 71, 1183-1189.

9. Dai, H.; Thostenson, E.T.; Schumacher, T. Processing and Characterization of a Novel Distributed Strain Sensor using Carbon Nanotube-Based Nonwoven Composites. Sensors 2015, $15,17728-17747$.

10. Yao, Y.; Glisic, B. Detection of Steel Fatigue Cracks with Strain Sensing Sheets Based on Large Area Electronics. Sensors 2015, 15, 8088-8108.

11. Naghashpour, A.; Van Hoa, S. A Technique for Real-Time Detecting, Locating, and Quantifying Damage in Large Polymer Composite Structures made of Carbon Fibers and Carbon Nanotube Networks. Structural Health Monitoring 2015, 14, 35-45.

12. Baltopoulos, A.; Polydorides, N.; Pambaguian, L.; Vavouliotis, A.; Kostopoulos, V. Damage Identification in Carbon Fiber Reinforced Polymer Plates using Electrical Resistance Tomography Mapping. J. Composite Mater. 2013, 47, 3285-3301. 
13. Baltopoulos, A.; Polydorides, N.; Pambaguian, L.; Vavouliotis, A.; Kostopoulos, V. Exploiting Carbon Nanotube Networks for Damage Assessment of Fiber Reinforced Composites. Composites Part B: Engineering 2015, 76, 149-158.

14. Loyola, B.R.; Briggs, T.M.; Arronche, L.; Loh, K.J.; La Saponara, V.; O’Bryan, G.; Skinner, J.L. Detection of Spatially Distributed Damage in Fiber-Reinforced Polymer Composites. Structural Health Monitoring 2013, 12, 225-239.

15. Loyola, B.R.; Saponara, V.; Loh, K.J.; Briggs, T.M.; O'Bryan, G.; Skinner, J.L. Spatial Sensing using Electrical Impedance Tomography. Sensors Journal, IEEE 2013, 13, 2357-2367. 16. Tallman, T.N.; Gungor, S.; Wang, K.; Bakis, C.E. Damage Detection Via Electrical Impedance Tomography in Glass Fiber/Epoxy Laminates with Carbon Black Filler. Structural Health Monitoring 2015, 14, 100-109.

17. Hou, T.; Loh, K.J.; Lynch, J.P. Spatial Conductivity Mapping of Carbon Nanotube Composite Thin Films by Electrical Impedance Tomography for Sensing Applications. Nanotechnology 2007, 18,315501 .

18. Loh, K.J.; Hou, T.; Lynch, J.P.; Kotov, N.A. Carbon Nanotube Sensing Skins for Spatial Strain and Impact Damage Identification. J. Nondestr. Eval. 2009, 28, 9-25.

19. Tallman, T.N.; Gungor, S.; Wang, K.; Bakis, C. Damage Detection and Conductivity Evolution in Carbon Nanofiber Epoxy Via Electrical Impedance Tomography. Smart Mater. Struct. 2014, 23, 045034.

20. Hou, T.; Lynch, J.P. Electrical Impedance Tomographic Methods for Sensing Strain Fields and Crack Damage in Cementitious Structures. J Intell Mater Syst Struct 2008.

21. Hallaji, M.; Pour-Ghaz, M. A New Sensing Skin for Qualitative Damage Detection in Concrete Elements: Rapid Difference Imaging with Electrical Resistance Tomography. NDT E Int. 2014, 68, 13-21.

22. Hallaji, M.; Seppänen, A.; Pour-Ghaz, M. Electrical Impedance Tomography-Based Sensing Skin for Quantitative Imaging of Damage in Concrete. Smart Mater. Struct. 2014, 23, 085001. 23. Karhunen, K.; Seppänen, A.; Lehikoinen, A.; Monteiro, P.J.; Kaipio, J.P. Electrical Resistance Tomography Imaging of Concrete. Cem. Concr. Res. 2010, 40, 137-145.

24. Dai, H.; Schumacher, T.; Thostenson, E. Carbon Nanotube-Based Sensing Composites for Structural Health Monitoring of Civil Infrastructure using Non-Woven Fabrics. In Safety, Reliability, Risk and Life-Cycle Performance of Structures and Infrastructures, Proceedings of the 11th International Conference on Structural Safety and Reliability (ICOSSAR), New York, NY, USA, 16-20 June, 2013; pp. 299.

25. Hu, N.; Karube, Y.; Arai, M.; Watanabe, T.; Yan, C.; Li, Y.; Liu, Y.; Fukunaga, H. Investigation on Sensitivity of a Polymer/Carbon Nanotube Composite Strain Sensor. Carbon 2010, 48, 680-687.

26. Yu, N.; Zhang, Z.H.; He, S.Y. Fracture Toughness and Fatigue Life of MWCNT/Epoxy Composites. Materials Science and Engineering: A 2008, 494, 380-384.

27. Li, C.; Thostenson, E.T.; Chou, T. Sensors and Actuators Based on Carbon Nanotubes and their Composites: A Review. Composites Sci. Technol. 2008, 68, 1227-1249.

28. Schumacher, T.; Thostenson, E.T. Development of Structural Carbon Nanotube-based Sensing Composites for Concrete Structures. J Intell Mater Syst Struct 2014, 25, 1331-1339. 29. Saafi, M. Wireless and Embedded Carbon Nanotube Networks for Damage Detection in Concrete Structures. Nanotechnology 2009, 20, 395502.

30. Ubertini, F.; Laflamme, S.; Ceylan, H.; Materazzi, A.L.; Cerni, G.; Saleem, H.;

D’Alessandro, A.; Corradini, A. Novel Nanocomposite Technologies for Dynamic Monitoring of 
Structures: A Comparison between Cement-Based Embeddable and Soft Elastomeric Surface Sensors. Smart Mater. Struct. 2014, 23, 045023.

31. Gao, L.; Chou, T.; Thostenson, E.T.; Zhang, Z.; Coulaud, M. In Situ Sensing of Impact Damage in Epoxy/Glass Fiber Composites using Percolating Carbon Nanotube Networks. Carbon 2011, 49, 3382-3385.

32. An, Q.; Rider, A.N.; Thostenson, E.T. Electrophoretic Deposition of Carbon Nanotubes Onto Carbon-Fiber Fabric for Production of Carbon/Epoxy Composites with Improved Mechanical Properties. Carbon 2012, 50, 4130-4143.

33. An, Q.; Rider, A.N.; Thostenson, E.T. Hierarchical Composite Structures Prepared by Electrophoretic Deposition of Carbon Nanotubes Onto Glass Fibers. ACS applied materials \& interfaces 2013, 5, 2022-2032.

34. Harikumar, R.; Prabu, R.; Raghavan, S. Electrical Impedance Tomography (EIT) and its Medical Applications: A Review. International Journal of Soft Computing Engineering 2013, 3, 2231-2307.

35. Brown, B. Electrical Impedance Tomography (EIT): A Review. J. Med. Eng. Technol. 2003, 27, 97-108.

36. Vauhkonen, M. Electrical Impedance Tomography and Prior Information. PhD Dissertation, Univeristy of Kuopio, Finland 1997.

37. Holder, D.S. Electrical Impedance Tomography: Methods, History and Applications.; CRC Press, 2004.

38. Silvera-Tawil, D.; Rye, D.; Soleimani, M.; Velonaki, M. Electrical Impedance Tomography for Artificial Sensitive Robotic Skin: A Review. Sensors Journal, IEEE 2015, 15, 2001-2016. 39. Tallman, T.N.; Gungor, S.; Wang, K.; Bakis, C. Tactile Imaging and Distributed Strain Sensing in Highly Flexible Carbon Nanofiber/Polyurethane Nanocomposites. Carbon 2015, 95, 485-493.

40. Somersalo, E.; Cheney, M.; Isaacson, D. Existence and Uniqueness for Electrode Models for Electric Current Computed Tomography. SIAM J Appl Math 1992, 52, 1023-1040.

41. Polydorides, N. Image Reconstruction Algorithms for Soft-Field Tomography. PhD Dissertation, University of Manchester Institute of Science and Technology, United Kingdom 2002.

42. Adler, A.; Guardo, R. Electrical Impedance Tomography: Regularized Imaging and Contrast Detection. Medical Imaging, IEEE Transactions on 1996, 15, 170-179.

43. Bera, T.K.; Nagaraju, J. A MATLAB-Based Boundary Data Simulator for Studying the Resistivity Reconstruction using Neighbouring Current Pattern. Journal of Medical Engineering 2013, 2013.

44. Polydorides, N.; Lionheart, W.R. A Matlab Toolkit for Three-Dimensional Electrical Impedance Tomography: A Contribution to the Electrical Impedance and Diffuse Optical Reconstruction Software Project. Measurement Science and Technology 2002, 13, 1871. 45. Persson, P.; Strang, G. A Simple Mesh Generator in MATLAB. SIAM Rev 2004, 46, 329-345. 46. Vauhkonen, P.J.; Vauhkonen, M.; Savolainen, T.; Kaipio, J.P. Three-Dimensional Electrical Impedance Tomography Based on the Complete Electrode Model. Biomedical Engineering, IEEE Transactions on 1999, 46, 1150-1160.

47. Richardson, M.; Wisheart, M. Review of Low-Velocity Impact Properties of Composite Materials. Composites Part A: Applied Science and Manufacturing 1996, 27, 1123-1131. 48. ASTM Standard. D7136/D7136M-05, Standard Test Method for Measuring the Damage Resistance of a Fiberreinforced Polymer Matrix Composite to a Drop-Weight Impact Event. West Conshohocken (PA): ASTM International 2005. 
49. Graham, B.; Adler, A. Objective Selection of Hyperparameter for EIT. Physiol. Meas. 2006, 27, S65.

50. Titman, D. Applications of Thermography in Non-Destructive Testing of Structures. NDT E Int. 2001, 34, 149-154.

51. Grinzato, E. State of the art and perspective of infrared thermography applied to building science. In Infrared Thermography Recent Advances and Future Trends.; C. Meola, Ed.; Bentham eBooks, 2012; pp. 200-229.

52. Kumar Vishwakarma, R.; Mahule, V.; Shahid, M. Fault Diagnosing of a High Density Electronic Card Employing Multiple Power Supplies using Infrared Thermography. Journal of Non destructive Testing \& Evaluation Vol 2008, 7.

53. Vincent, L.; Marie-France, D.; Bovic, K.; Pierre, D. Evaluation of the Performance of Infrared Thermography for on-Line Condition Monitoring of Rotating Machines. Engineering 2011, 2011.

54. Meola, C.; Carlomagno, G.M.; Squillace, A.; Vitiello, A. Non-Destructive Evaluation of Aerospace Materials with Lock-in Thermography. Eng. Failure Anal. 2006, 13, 380-388.

55. Menaka, M.; Bagavathiappan, S.; Venkatraman, B.; Jayakumar, T.; Raj, B. Characterisation of Adhesively Bonded Laminates using Radiography and Infrared Thermal Imaging Techniques. Insight-Non-Destructive Testing and Condition Monitoring 2006, 48, 606-612.

56. Bagavathiappan, S.; Lahiri, B.; Saravanan, T.; Philip, J.; Jayakumar, T. Infrared

Thermography for Condition Monitoring-a Review. Infrared Phys. Technol. 2013, 60, 35-55. 57. Boyle, A.; Adler, A. The Impact of Electrode Area, Contact Impedance and Boundary Shape on EIT Images. Physiol. Meas. 2011, 32, 745. 